\title{
Method Development and Validation of Ephedrine and Pseudoephedrine in Horse Urine by Liquid Chromatography-Tandem Mass Spectrometry
}

\author{
Ashok Kumar Maurya1', Puran Lal Sahu1, Sakshi Jethi', Himank Gosain², Kapendra Sahu1,* \\ ${ }^{1}$ National Dope Testing Laboratory, Ministry of Youth Affairs and Sports (MYAS), Government of India, New Delhi, INDIA. \\ ${ }^{2}$ Department of Chemistry, Manav Rachna University, Haryana, INDIA.
}

\begin{abstract}
Background: A comprehensive, fast and precise method for the quantification of ephedrine and pseudoephedrine in horse urine was established and validated utilizing Liquid Chromatography-Tandem Mass Spectrometry (LC-MS/MS) followed by the necessities of Association of Official Racing Chemists (AORC), Federation of Equine International (FEI) and International Council for Harmonization (ICH) guidelines. Methodology: The processing of samples was performed by de conjugation accompanied by enzymatic hydrolysis, Solid Phase Extraction (SPE) procedure using HLB cartridges. Results: The total chromatographic run time of this method was $7.0 \mathrm{~min}$ and found linear over the concentration range of $4-40 \mathrm{ng} / \mathrm{mL}$ with a correlation of coefficient $\left(r^{2}\right)$ of 0.99 . The intraday and inter-day assay precision of ephedrine were extended from 1.64 to $2.86 \%$ and 1.61 to $3.12 \%$, respectively. In context of pseudoephedrine, the intraday and interday assay precision were ranged from 1.55 to $3.26 \%$ and 1.11 to $2.60 \%$, respectively. The absolute percent recovery (\%) was found to be $85 \%$ for Ephedrine and $90 \%$ for Pseudoephedrine. The percent recovery was adequate to discriminate and quantitate the Ephedrine and Pseudoephedrine at or below the level prescribed by AORC Proficiency Testing (PT) list ie $20 \mathrm{ng} / \mathrm{ml}$ for equine urine sample. The LOD and LOO were found 2 and $4 \mathrm{ng} / \mathrm{ml}$ respectively for Ephedrine and Pseudoephedrine. Conclusion: A comprehensive and precise method for quantitative estimation and diastereomeric differentiation of Ephedrine and Pseudoephedrine by LC-MS/MS was well established and validated according to AORC, $\mathrm{FEI}$ and $\mathrm{ICH}$ guidelines. This strategy may be additionally utilized for remedial medication checking purposes.
\end{abstract}

Key words: Ephedrine, Pseudoephedrine, ICH Guidelines, Doping control, Mass spectrometry, AORC, Validation.

\section{INTRODUCTION}

Most equine doping samples are still urine, which offers some essential benefits in comparison to blood. Most analyses can be found in higher concentration resulting in a longer detection time. Furthermore, metabolites can be detected to fortify a positive result and to prove the passage of a drug through the horse urine. ${ }^{1-3}$ Ephedrine is a drug belong to stimulant category. It is widely used to counteract the effects of anesthesia and also in therapies for the treatment of asthma and narcolepsy. It has been proven that Ephedrine has common side effects i.e heart attack; dizziness; high blood pressure; headache; nausea; nervousness; fast heart rate; tremor; loss of appetite; seizures; stroke; restlessness; trouble sleeping; stomach irritation, inability to urinate psychosis and abuse. Ephedrine has the high potential to boost horse and human athletic performance. ${ }^{1}$

Pseudoephedrine is a stimulant and sympathomimetic drug. Pseudoephedrine overdose can result in a mainly irregular heartbeat; sweating; hyperactivity; mydriasis; trouble sleeping; tachycardia; hallucinations;
Submission Date: 17-03-2020; Revision Date: 29-06-2020; Accepted Date: 13-08-2020

DOI: 10.5530/ijper.54.3s.167 Correspondence: Dr. Kapendra Sahu National Dope Testing Laboratory, Ministry of Youth Affairs and Sports (MYAS), Government of India, New Delhi-110003, INDIA.

Phone: +91-011-24368850 E-mail: kapendra@gmail. com

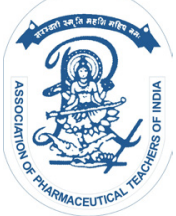

www.ijper.org 
hypertension; sinus arrhythmias; headache; anxiety; tremors; hyperthermia; head bobbing, hiding; psychiatric and symptoms like paranoia. Pseudoephedrine shares the pharmacologic properties of ephedrine but has less potent CNS-stimulating effects. ${ }^{2,3}$

Ephedrine and Pseudoephedrine are obtained from the plant ephedra silica and other members of the genus ephedra. Ephedrine has similar in molecular structure to phenylpropanolamine, methamphetamine and epinephrine (adrenaline). However, the effects of ephedrine are much less potent and longer acting than amphetamines. Ephedrine exhibits optical isomerism and has two chiral centers, giving rise to four stereoisomers, Pseudoephedrine is a stereoisomer of ephedrine that is used as a nasal decongestant. The decongestant effect of pseudoephedrine in equine was described in $1927 .{ }^{4}$

Ephedrine and Pseudoephedrine may be misused because of their performance enhancing potential and thus potential misuse should be controlled by racing laboratories. Ephedrine and Pseudoephedrine can be quantified in urine to screen the possible abuse in horses. The anti-doping rules for Federation of Equine International (FEI) ban the use of Ephedrine and Pseudoephedrine in horse sport. The concentration limit of Ephedrine and Pseudoephedrine in the proficiency testing program is defined $20 \mathrm{ng} / \mathrm{ml}$ in equine urine by Association of Official Racing Chemists (AORC). ${ }^{5}$

In recent years, detection methods have been improved, resulting in the detection of prohibited substances including Ephedrine and Pseudoephedrine by gas chromatography nitrogen phosphorous detector (GC-NPD), gas chromatography-mass selective detector (GC-MSD), ion mobility MS (IM-MS) and liquid chromatography-tandem mass spectrometer (LC-MS/MS). While the GC-MSD has long been the suitable method in forensic testing and anti-doping field, LC-MS/MS in positive ESI has become a more common and sensitive technique for quantification of Ephedrine and Pseudoephedrine in horse urine in comparison with conventional GC-MSD. ${ }^{6-13}$

To our knowledge, information on separation methods for ephedrine and pseudoephedrine detectability and validation study while following AORC, FEI \& ICH Guideline in horse urine matrix using LC-MS/MS was not reported. The structure of ephedrine and pseudoephedrine is shown in Figure 1(a) and Figure 1(b), respectively. A LC-MS/MS method was developed and validated to detect and quantify the Ephedrine and Pseudoephedrine in horse urine samples. Horse urine samples were analyzed using the developed method to demonstrate the applicability of the method as per ICH, FEI and AORC guidelines. ${ }^{5,14,15}$

\section{MATERIALS AND METHODS}

\section{Material, Chemical and Standard Solution}

Certified Reference Material (CRM) of Ephedrine, Pseudoephedrine and D3-Ephedrine were procured from National Measurement Institute (NMI), Australia and Toronto Research Chemicals (TCS), Canada. Sodium Bicarbonate $\left(\mathrm{NaHCO}_{3}\right)$, Potassium carbonate $\left(\mathrm{K}_{2} \mathrm{CO}_{3}\right)$, Ammonium sulphate $\left(\mathrm{NH}_{4}\right)_{2} \mathrm{SO}_{4}$, Potassium Phosphate $\left(\mathrm{K}_{3} \mathrm{PO}_{4}\right)$, Methanol $\left(\mathrm{CH}_{3} \mathrm{OH}\right)$, Tert-Butyl methyl ether $\left.\left(\mathrm{CH}_{3}\right)_{3} \mathrm{COCH}_{3}\right)$, Formic acid $(\mathrm{HCOOH})$, Hydrochloric acid $(\mathrm{HCl})$, Sodium hydroxide $(\mathrm{NaOH})$ were purchased from E Merck Ltd., India and $\beta$-Glucoronidase enzyme (E. coli) was supplied by Roche, Germany.

\section{Instrumentation and chromatographic conditions}

LC-MS/MS Analysis was performed using Thermo Scientific TSQ Triple Quadrupole Mass spectrometer Dionex Ultimate 3000 UHPLC Plus equipped with Automatic Liquid Sampler (ALS). Chromatographic separation was performed on a Inertsil ODS-3, C-8 column $(4.6 \mathrm{~mm} \times 50 \mathrm{~mm}, 3 \mu \mathrm{m})$ with a gradient elution of $0.1 \%$ formic acid in water (solvent $\mathrm{A}$ ) and Methanol (solvent B).

Aliquots were prepared by taking $5 \mathrm{ml}$ of samples in test tube. Three gram of ammonium sulphate was added in each test tube and dissolved well on cyclo mixer. Then all the samples were centrifuged for $10 \mathrm{~min}$ and matrix was cleaned. Cleaned samples were taken in different test tubes. $6.0-6.5 \mathrm{pH}$ was maintained by adding $1 \mathrm{M}$ $\mathrm{HCl} / \mathrm{NaOH}$ in urine sample and any change was resisted in $\mathrm{pH}$ by adding $1 \mathrm{ml}$ phosphate buffer. Ephedrine and pseudoephedrine mixture was spiked in different quantities into horse urine samples. Then $100 \mu$ Internal Standard (D3-Ephedrine) of $1 \mu \mathrm{g} / \mathrm{ml}$ concentration was added into spiked urine samples. The $50 \mu$ enzyme $\beta$-glucoronidase from $E$. coli was added in all urine samples and mixed it properly, then samples were placed in incubator at 60 degree celsius temperature for $1 \mathrm{hr}$. Meanwhile, all the samples were placed in incubator \& prequiliberate HLB cartridges with $2 \mathrm{ml}$ methanol, $2 \mathrm{ml}$ water and $2 \mathrm{ml}$ of $0.1 \mathrm{M}$ phosphate buffer $(\mathrm{pH}$ 6.0). After incubation all the urine samples were again centrifuged for $5 \mathrm{~min}$. All the urine samples were then loaded in HLB cartridges. Samples in HLB cartridge were washed with $3 \mathrm{ml}$ water, $1 \mathrm{ml}$ of $5 \%$ methanol and $0.1 \mathrm{M}$ phosphate buffer. Then final elution was done by using $3 \mathrm{ml}$ methanol. After elution, all the eluted 
methanol was dried using $\mathrm{N}_{2}$ evaporator. All the dried residues were reconstituted with $100 \mu \mathrm{l}$ mobile phase $(0.1 \%$ Formic acid + methanol, $50: 50)$ and collected in vials and injected at LC-MS/MS.

Mass spectrometric analysis was performed on a triple quadrupole mass spectrometer operated in the multiple reaction monitoring (MRM) mode with the transitions of $166 \rightarrow 77, \quad 166 \rightarrow 115,166 \rightarrow 117, \quad 166 \rightarrow 133$ and $166 \rightarrow 148$ for Ephedrine/Pseudoephedrine with different retention times and $169 \rightarrow 150.9$ for D3Ephedrine as shown in Table 1. LC-Quan-3.0 (Software) was used for calculation of concentration for Ephedrine and Pseudoephedrine.

The Ion transfer tube temperature was kept at $350^{\circ} \mathrm{C}$, while vaporizer temperature was $400^{\circ} \mathrm{C}$ in Electron Impact (EI) mode, with a solvent delay of 2 min. Data were acquired in the Multiple Reaction Monitoring (MRM) mode, by using this diagnostic transition of the analyses (Ephedrine and Pseudoephedrine) as shown in Table 1.

Molecular Formula: $\mathrm{C}_{10} \mathrm{H}_{15} \mathrm{NO}$

Molecular Weight: $165.23 \mathrm{~g} / \mathrm{mol}$

The method was established as per the requirements of ICH, FEI and AORC guidelines. To develop the linearity and range, a stock solution containing $1 \mathrm{mg} /$ $\mathrm{ml}$ drug in ethanol was diluted to yield solutions in the concentration range $4-40 \mathrm{ng} / \mathrm{ml}$ and keeping the injection volume constant $(5 \mu \mathrm{L})$. To assess precision, five injections of five different QC concentrations (6, 8, 20 and $25 \mathrm{ng} / \mathrm{ml}$ ) were made on the same day and intra-day precision was determined as relative standard deviation. These studies were also repeated on different days to determine inter-day precision. Accuracy was evaluated by fortifying a mixture of solution with three known concentrations of the drugs and recovery of the added drugs were evaluated. The specificity of the method for the drugs were established by analyzing horse urine samples collected from twelve different horse to investigate the potential interferences at the LC peak region for analysts and Internal Standard (IS) using the proposed extraction procedure and chromatographic-MS conditions. The Limits of
Detection (LOD) and Limit of Quantification (LOQ) were determined experimentally, by analysis of samples spiked with decreasing concentrations of the analyses. LOD was defined as the concentration yielding a signalto-noise ratio of 3. LOQ was calculated as the smallest concentration of analytic that could be measured with a signal-to-noise ratio of 10 .

\section{Recovery}

Recoveries of the Ephedrine and Pseudoephedrine were determined for horse urine accompanied by the analysis of six different urine samples spiked at $20 \mathrm{ng} / \mathrm{ml}$.

\section{RESULTS AND DISCUSSION}

\section{Method development and optimization}

The optimization and quantification of the mass spectrometric condition were carried out in a multistep procedure. Initially, a full scan spectrum was acquired for each compound. Based on the full scan spectra followed by analysis of base peak, a suitable precursor ion was selected. Some injection was also run to collect the appropriate product ion succeeded byproduct scan mass spectra using different collision energies (CEs). An appropriate product ion and optimization of the collision energy was then carried out on both reference standard and extract from spiked horse urine samples. The Multiple Reaction Monitoring (MRM) diagnostics ions are shown in Table 1.

The experimental conditions for LC-MS/MS method ie., composition of mobile phase, flow rate and multiple reaction monitoring (MRM) for detection

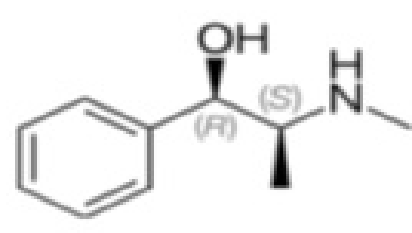

(a)

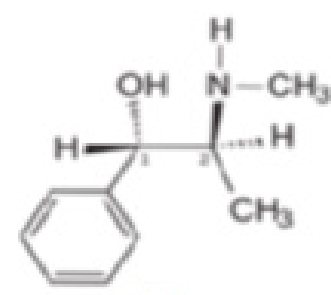

(b)
Figure 1: Structure of (a) (1R,2S)-(-)-Ephedrine and (b) (1S,2S)-(+)-Pseudoephedrine.

\begin{tabular}{|c|c|c|c|c|c|}
\hline \multicolumn{5}{|c|}{ Table 1: Diagnostic MRM transition for Ephedrine, Pseudoephedrine and ISTD. } \\
\hline Name of Analyte & $\begin{array}{c}\text { Precursor } \\
(\mathbf{m} / \mathbf{z})\end{array}$ & Product ion & RT (Minutes) & MRM & CE \\
\hline Ephedrine/ & 166 & 77 & $4.86 / 5.79$ & $166>77$ & 35 \\
Pseudoephedrine & & 115 & & $166>115$ & 29 \\
& & 117 & & $166>117$ & 21 \\
& & 133 & & $166>133$ & 22 \\
\hline D3-Ephedrine & 169 & 148 & & $166>148$ & 20 \\
\hline
\end{tabular}


were augmented to provide precise, accurate and reproducible results for the simultaneous determination of Ephedrine and Pseudoephedrine in horse urine.

The operational strategy for stability of the method was tried with spiked samples of $20 \mathrm{ng} / \mathrm{ml}$ in five different specific gravity (SG) urine specimens with $5 \mathrm{uL}$ injection volume, comparing to $20 \mathrm{ng} / \mathrm{mL}$ of standard norms. The reason for the test was to assess the operational steadiness against the electrospray ionization (ESI) source contamination by urine samples after SPE clean-up. Figure 2 shows the representative chromatogram of ephedrine and pseudoephedrine with good resolution. Furthermore, Figure 3 shows the representative chromatogram D3-ephedrine, which was used as an internal standard. Figure 4 depicts the absence of any peak in drug free urine (DFU) at the retention time of standards, which represents the absence of co-elution and carry over during the injection of matrix. Based on these outcomes, it is possible to carry out analysis of horse urine samples after SPE clean-up for repeatable result by the high sensitivity LC-MS/MS technique with a low injection volume of $5 \mathrm{uL}$.

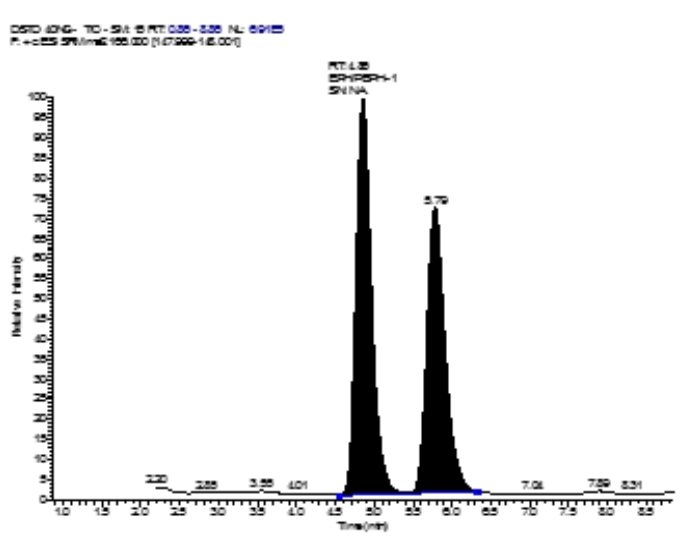

Figure 2: Representative MRM ion Chromatogram of Ephedrine and Pseudoephedrine in Horse Urine.

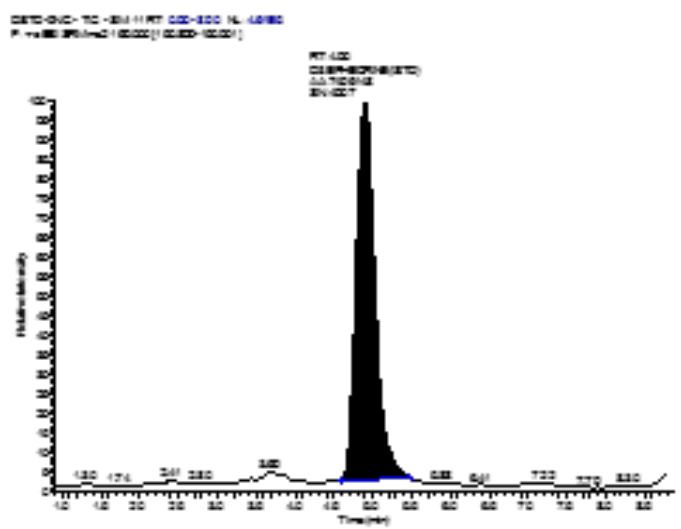

Figure 3: Representative MRM ion Chromatogram for D3Ephedrine in Horse Urine
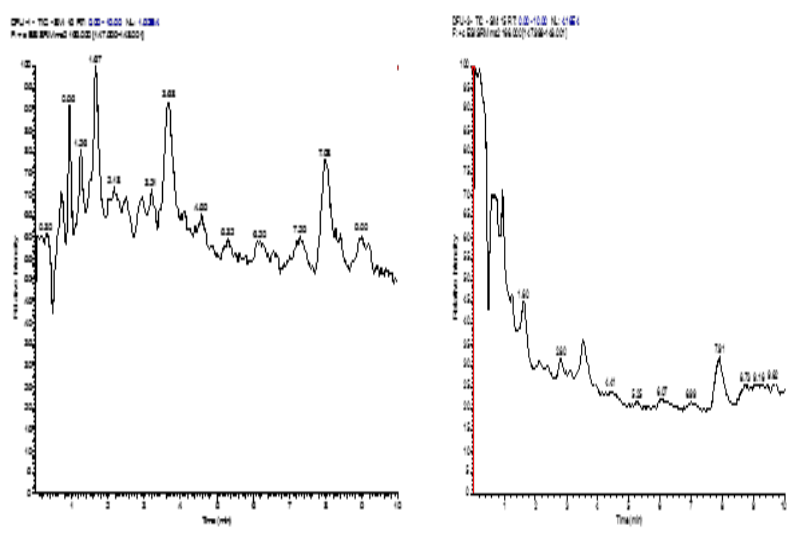

Figure 4: Representative MRM ion Chromatogram for HorseDrug Free Urine (DFU).

The electrospray ionization was carried out in positive ionization mode for the analytical standards and its internal standard (IS). The accompanying instrument parameters for ionization were utilized: spray voltage: 4500 volt, Sweep gas: 2 Arb, Sheath gas: 50 Arb and Aux gas: 20 Arb.

\section{Validation}

The calibration curve was plotted utilizing five linear points viz., $4-40 \mathrm{ng} / \mathrm{mL}$. The linearity standard curve represented a reliable reproducibility over the standard concentrations across the linear range. The response of calibration curve was organized by determining the best fit of peak-area ratios (peak area analyte/peak area IS) versus concentration and best fitted to the $y=m x+c$ using weighing factor $(1 / \mathrm{X})$. The regression equation obtained were $y=0.197 x+0.0002$ and the correlation coefficient, $r^{2}$, for the equation was 0.98 . The precision and Accuracy were studied for intraday and interday samples ranged from $0.75-1.7 \%$ and $87-111 \%$, respectively, indicating the method was sufficiently precise. The measures values on intraday and inter-day were found to be within the expected limits as given in Table 2. The percentage recovery for Ephedrine and Pseudoephedrine was found to be $85 \%$ and $90 \%$ respectively at $20 \mathrm{ng} / \mathrm{ml}$ spiking level. The percentage recovery was sufficient to reliable identify of Ephedrine and Pseudoephedrine in horse urine at or below the level prescribed by AORC proficiency testing list-2019 i.e $20 \mathrm{ng} / \mathrm{ml}$. The LOD and LOQ was measured to be 2 and $4 \mathrm{ng} / \mathrm{ml}$, respectively.

\section{CONCLUSION}

A comprehensive, fast and precise LC-MS/MS method for quantitative determination of Ephedrine and Pseudoephedrine was established and validated followed by ICH, FEI and AORC guidelines. This strategy 


\begin{tabular}{|c|c|c|c|c|c|c|}
\hline \multirow{2}{*}{$\begin{array}{c}\text { Quality } \\
\text { Controls (ng/ } \\
\text { ml) }\end{array}$} & \multicolumn{3}{|c|}{ Ephedrine } & \multicolumn{3}{|c|}{ Pseudoephedrine } \\
\hline & $\begin{array}{c}\text { Mean } \pm S D \\
\quad(n=5)\end{array}$ & $\begin{array}{c}\text { Accuracy } \\
(\%)\end{array}$ & $\begin{array}{c}\text { Precision } \\
\text { (\%) }\end{array}$ & $\begin{array}{c}\text { Mean } \pm S D \\
(n=5)\end{array}$ & $\begin{array}{c}\text { Accuracy } \\
(\%)^{\mathrm{a}}\end{array}$ & $\begin{array}{c}\text { Precision } \\
(\%)^{\mathrm{b}}\end{array}$ \\
\hline \multicolumn{7}{|c|}{ Intraday precision } \\
\hline 6 & $5.456 \pm 0.111$ & 90.93 & 2.035 & $5.602 \pm 0.113$ & 93.37 & 2.025 \\
\hline 8 & $7.654 \pm 0.126$ & 95.68 & 1.644 & $7.498 \pm 0.245$ & 93.73 & 3.264 \\
\hline 20 & $19.732 \pm 0.333$ & 98.66 & 1.687 & $19.750 \pm 0.307$ & 98.75 & 1.554 \\
\hline 25 & $25.322 \pm 0.726$ & 101.29 & 2.864 & $25.328 \pm 0.520$ & 101.31 & 2.054 \\
\hline \multicolumn{7}{|c|}{ Inter day precision } \\
\hline 6 & $5.576 \pm 0.174$ & 92.93 & 3.123 & $5.854 \pm 0.152$ & 97.57 & 2.603 \\
\hline 8 & $7.670 \pm 0.124$ & 95.88 & 1.615 & $7.710 \pm 0.153$ & 96.38 & 1.986 \\
\hline 20 & $20.308 \pm 2.737$ & 101.54 & 2.737 & $20.360 \pm 2.63$ & 101.80 & 2.635 \\
\hline 25 & $24.754 \pm 1.737$ & 99.01 & 1.771 & $25.804 \pm 0.289$ & 103.22 & 1.114 \\
\hline
\end{tabular}

a Determined as (mean concentration/nominal concentration) $\times 100$

${ }^{\mathrm{b} C}$ Calculated as \% RSD (Standard deviation/mean).

was specific, accurate and reproducible for Ephedrine and Pseudoephedrine. The estimated run time of the developed method was $7 \mathrm{~min}$ at gradient program. This current method would get enhanced by combining the triple quadrupole with Liquid Chromatography, thereby can greatly improve the detection capabilities and minimizing false detection of target substance in complex horse urine matrix. This method was efficiently applied for screening and confirmation analysis for the testing of horse dope samples. Further, the extension of this work would be on the differentiation of results of Ephedrine and Pseudoephedrine analysis in horse urine due to presence of confounding/interfering factors. The higher sensitivity with shorter run time contains low sample volume, the better LOD/LOQ, the less tedious cleaning up and sample preparation procedure make this preferred analytical procedure using LC-MS/ MS methodology.

\section{ACKNOWLEDGEMENT}

The author wishes to acknowledge the Ministry of Youth Affairs and Sports, Govt. of India for their continuous support.

\section{CONFLICT OF INTEREST}

The authors declare no conflict of interest, financial or otherwise.

\section{ABBREVIATIONS}

AORC: Association of Official Racing Chemists; ICH: International Council for Harmonization, FEI: Federation of Equine International; HPLC: High Performance Liquid Chromatography; GC-MS: Gas Chromatography- mass spectrometry; SPE: Solid Phase Extraction; LC-MS: Liquid chromatography mass spectrometry; ESI: Electrospray Ionization; MRM: Multiple Reaction Monitoring; RT: Retention time; Arb: Arbitrary unit; CE: Collision Energy; HLB: Hydrophilic Lipophilic Balance.

\section{REFERENCES}

1. Gabriel RA, Andressa MS, Raquel M, Monica C, Francisco R, Henrique $M G$, et al. Fast ephedrine quantification by gas chromatography mass spectrometry. J Braz Chem Soc. 2018;29(12):2514-21.

2. Hodges $K$, Hancock $S$, Currell $K$, Hamilton $B$, Jeukendrup $A E$. Pseudoephedrine enhances performance in 1500-m runners. Med Sci Sports Exerc. 2006;38(2):329-33.

3. Minamizawa K, Goto H, Shimada Y, Terasawa K, Haji A. Effect of d-Pseudoephedrine on cough reflex and its mode of action in guinea pigs. Journal of Pharmacological Sciences. 2006;102(1):136-42.

4. https://pubchem.ncbi.nlm.nih.gov

5. www.aorc-online.org

6. Ahi S, Sahu K, Nasare M, Singh S, Beotra A, Jain S. Quantitative estimation of alcohol marker ethyl glucuronide (EtG) in human hair by LC-MS/MS: An Application Towards Doping Control and Forensic Science. Current Chromatography. 2018;5(2):112-6.

7. Sahu K, Patel P, Karthikeyan C, Trivedi P. The ICH Guidance in Practice: Stress degradation studies on irbesartan and development of a validated stability-indicating UPLC Assay. Acta Chromatographica. 2010;22(2):189205. 
Jain S, Sahu PL, Raj S, Shrivastava A, Maurya AK, Tonk R, et al. Method development and validation for detection of procaterol in human urine using gas chromatography-tandem mass spectrometry. Indian Journal of Pharmaceutical Education and Research. 2019;53(4):669-77.

9. Eenoo PV, Delbeke FT, Roels K, DeBacker P. Simultaneous quantitation of ephedrines in urine by gas chromatography-nitrogen-phosphorus detection for doping control purposes. J Chromatogr B Biomed Sci Appl. 2001;760(2):255-61.

10. Zhanliang W, Jianghai L, Yinong Z, Ye T, Hong Y, Youxuan X. Applications and challenges in using LC-MS/MS assays for quantitative doping analysis. Bioanalysis: 2016;8(12):1307-22.
11. Lokhnauth JK, Snow NH. Solid phase micro-extraction coupled with ion mobility spectrometry for the analysis of ephedrine in urine. J Sep Sci. 2005;28(7):612-8.

12. Deventer K, Pozo OJ, Eenoo PV, Delbeke FT. Development and validation of an LC-MS/MS method for the quantification of ephedrines in urine. J Chromatogr B Analyt Technol Biomed Life Sci. 2009;877(4):369-74.

13. Stout PR, Klette KL, Horn CK. Evaluation of ephedrine, pseudoephedrine and phenylpropanolamine concentrations in human urine samples and a comparison of the specificity of DRI amphetamines and Abuscreen online (KIMS) amphetamines screening immunoassays. J Forensic Sci. 2004;49(1):160-4.

14. https://www.ich.org

15. https://inside.fei.org/fei/cleansport/ad-h/prohibited-list

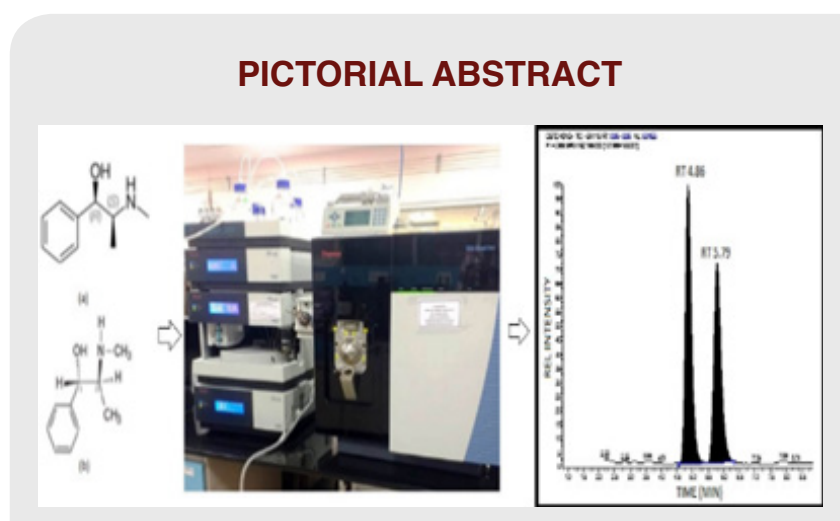

\section{SUMMAY}

Liquid Chromatography-Tandem Mass spectrometry obviously has a crucial and focal role in the screening and confirmation analysis of medications of misuse at present and later on. The improvement toward more sensitive methodology will always a continuous process to avoid false reporting accompanied by reliable results. Based on the utility of this methodology, the stereoisomers can be easily discriminated, this analytical method offers substantial regulatory and scientific advantages over the conventional urine testing methods.

Cite this article: Maurya AK, Sahu PL, Jethi S, Gosain H, Sahu K. Method Development and Validation of Ephedrine and Pseudoephedrine in Horse Urine by Liquid Chromatography-Tandem Mass Spectrometry. Indian J of Pharmaceutical Education and Research. 2020;54(3s):s671-s676. 